\title{
Ceramic Biomaterials: What are their Biological Implications?
}

ISSN: 2637-8078

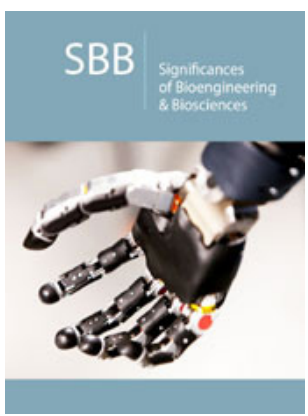

*Corresponding author: Alison TysonCapper, Translational and Clinical Research Institute, Faculty of Medical Sciences, Newcastle University, UK

Submission: 阱January 20, 2021

Published: 監February 08, 2021

Volume 4 - Issue 4

How to cite this article: Jamieson $\mathrm{S}$ Anjum S, Kirby J, Alison Tyson-Capper. Ceramic Biomaterials: What are their Biological Implications? Significances of Bioengineering \& Biosciences .4(4). SBB. 000592. 2021.

DOI: 10.31031/SBB.2021.04.000592

Copyright@ Alison Tyson-Capper, This article is distributed under the terms of the Creative Commons Attribution 4.0 International License, which permits unrestricted use and redistribution provided that the original author and source are credited.
Jamieson S, Anjum S, Kirby J and Alison Tyson-Capper*

Faculty of Medical Sciences, Newcastle University, UK

\section{Abstract}

Total Joint Arthroplasty (TJA) is an effective treatment for end-stage osteoarthritis. In 2019 there were 95,677 primary total hip arthroplasty (THA) procedures performed in England, Wales and Northern Ireland and this is set to increase with our ageing population and rising obesity. As implants used in THA last on average $79.9 \%$ at 20 years, many patients require revision surgery which is associated with increased risks of infection, venous thromboembolism and mortality. Ceramics have become an increasingly popular implant bearing surface and their use has been increasing annually since 2008, with ceramic-on-polyethylene implants accounting for 37.8\% of all primary THA performed in England, Wales and Northern Ireland. This is due to their reported properties of high wear resistance rate and being biologically inert. This brief review will cover examples of reported mechanical failure leading to component fracture, wear and audible hip squeaking in patients with ceramic implants. The ceramic wear debris in turn can stimulate a host inflammatory response, leading to osteolysis, aseptic loosening and pseudotumour formation. As ceramic use in hip arthroplasty increases, further understanding of the mechanical and biological mechanisms driving implant failure is required to improve implant longevity for patients and reduce revision surgery rates.

Keywords: Ceramic biomaterials; Biological implications; Osteoarthritis; Surgery; Polyethylene implants; Analgesia; Physiotherapy; Venous thromboembolism;High wear resistance; Corrosion

Abbreviations: OA: Osteo Arthritis; TJA: Total Joint Arthroplasty; TLR4: Toll-Like Receptor 4; UHMWPE: Ultra-High-Molecular-Weight-Polyethylene; PBMCs: Peripheral Blood Mononuclear Cells

\section{Osteoarthritis}

Osteoarthritis (OA) is a metabolically active condition characterised by loss of protective hyaline cartilage on joint surfaces. The subsequent contact of articulating bone surfaces within joints leads to subchondral sclerosis, cyst formation as well as osteophytes. This manifests clinically as joint pain, stiffness, swelling and loss of function for $10 \%$ of men and $18 \%$ of women aged over 60 years[1]. There are no licenced medications to limit OA progression and when symptoms are refractory to conservative treatments such as analgesia and physiotherapy, total joint arthroplasty (TJA) is indicated.

\section{Total joint replacement}

TJA is an effective treatment in end-stage OA using biomaterials to replace diseased joint surfaces and in 2019 there were 95,677 primary total hip replacements performed in England, Wales and Northern Ireland with osteoarthritis being indicated in $91.5 \%$ of cases[2]. This number is set to increase with our ageing population and rising obesity. As implants last on average for $78.9 \%$ at 20 years there is an urgent need to understand mechanisms driving implant failure, as revision surgery is associated with increased morbidity, mortality, venous thromboembolism rates and poorer subsequent function[3].The most common combination of hip replacement bearing surfaces are a metal alloy femoral head component articulating with a polyethylene acetabular cup, which has been in use since the development of the primary total hip replacement by Sir John Charnley in the 1960s[4]. However, metal-onpolyethylene (MoP) implants have been implicated in adverse reactions to debris as a result of phagocytosis of particulate debris by peri-implant macrophages, leading to osteolysis[5]. Ceramics have become increasingly popular as an alternative bearing surface in femoral head components as compared to MoP, as they are considered to be more biologically inert and have high wear resistance rates, in addition to reduced metal ion release from taper corrosion, which is observed in metal implants complicated by aseptic, lymphocyte-dominated vasculitis lesion (ALVAL) $(6,7)$. Given the increasing proportion of use of ceramic-on-polyethylene (CoP) 
in both cemented and uncemented implants annually since 2008 (Figure 1) and the projected increase in total hip arthroplasty cases, it is important to understand the mechanical and biological mechanisms of ceramic implant failure.

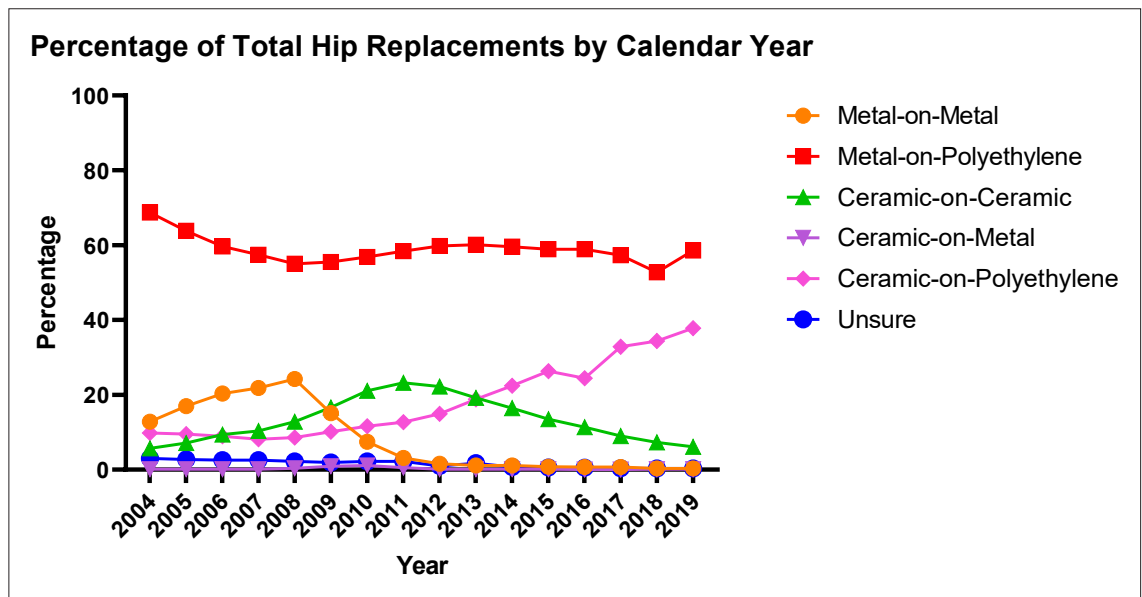

Figure 1: The use of ceramic-on-polyethylene for primary total hip replacement has consistently increased in usage year-on-year while metal-on-polyethylene prostheses remain the most popular in England and Wales. Metal-on-metal implant usage peaked in 2008 but declined in popularity and now account for approximately $0.3 \%$ of all primary hip replacements. Ceramic-on-ceramic implants, similarly to metal-on-metal, also saw an increase in popularity which peaked in 2011 but has been declining since. Data is from The National Joint Registry 2020 [2].

\section{Failure}

Initial case reports identified a pattern of mechanical implant failure due to fracture of ceramic-polyethylene sandwich liner acetabular components in addition to worn ceramic head components in hip arthroplasty implants[8,9]. However, there have been a number of case reports detailing the failure of a ceramic implants due to a variety of reasons. One case report detailed the failure of a ceramic-on-ceramic ( $\mathrm{CoC}$ ) implant which had been indicated for primary osteoarthritis 5-years prior in a 68-years old patient who initially presented with a squeaking in their hip which could be heard up to 25 metres away[10]. Analysis following revision surgery demonstrated that the acetabular cup had completely worn through and the femoral head had broken, this wear equated to $10 \mathrm{~mm}^{3}$ /day for 1 year. Whilst this is an extreme example, the incidence of hip squeaking ranges from $<0.5$ to $11 \%$ following total hip arthroplasty and although it is usually not associated with functional impairment or pain, it may be indicative of serious prosthetic complications [4]. If hip squeaking following CoC total hip arthroplasty occurs it may cause failure of the implant and this will need to be taken into account in the future should these implants become more popular. Additionally, it is likely that overtime the accumulation of ceramic debris could cause a proinflammatory response similar to that seen in failed metal-onmetal (MoM) cobalt-chromium hip implants.

Pro-inflammatory pseudotumours are a benign mass of tissue often associated with failure of cobalt-chromium implants and the subsequent accumulation of cobalt debris. However, a case report by Campbell et al.[11], described a 54-years old patient who had received a $\mathrm{CoC}$ hip replacement 6-year prior, presenting with pain and grinding in their hip when moving[11]. Interestingly, upon radiological investigation a pseudotumour measuring $5 \times 4 \times 3 \mathrm{~cm}$ was found which when excised and stained using haematoxylineosin, consisted a mixed immune cellular infiltrate including both innate and adaptive immune cells. This is a clear demonstration of the potential pro-inflammatory and immunomodulatory effects of ceramic wear and highlights a clear similarity between the immunological effects of metals such as cobalt-chromium and reportedly inert ceramics. These case reports show a pattern of failure which may vary in origin from fracture to extensive wear which results in a pro-inflammatory response and the need for costly revision surgery.

\section{Biological mechanisms}

Previous research has implicated toll-like receptor 4 (TLR4) in the activation of monocyte and endothelial cell lines in response to cobalt ions[12,13]. However, at present a limited repertoire of literature has been published investigating the potential biological implications of ceramic biomaterials. Early evidence detailing biopsies taken from patients with $\mathrm{CoC}$ implants has shown macrophage infiltration detected using immunohistochemical staining[14] which suggests that ceramics are not inert as initially presumed. An in-vitro study published by Germain et al. [15], compared the cytotoxicity of clinically relevant cobalt-chromium to alumina ceramic wear particles and concluded that alumina particles which were generated in a hip simulator were mildly cytotoxic, however, the cobalt-chromium particles used in this study were much more cytotoxic and therefore of more focus throughout the study [15]. 
Mouse macrophage models have also been utilised and studies have demonstrated the ability of alumina particles in combination with ultra-high-molecular-weight-polyethylene (UHMWPE) to increase TNF $\alpha$ secretion as well as inducing apoptosis [16]. These findings agreed with a study published by Sterner et al.[17], which demonstrated a four-fold increase in TNF $\alpha$ protein secretion by THP-1 macrophage cells upon stimulation with alumina oxide particles similar to that used in total joint replacement implants, however, zirconium oxide particles which are also used in ceramic prostheses demonstrated no effects on the secretion of TNF $\alpha$ in this study [17]. Moreover, Bertrand et al. [18], also demonstrated that peripheral blood mononuclear cells (PBMCs) cultured on ceramic surfaces containing both alumina and zirconium experienced significant secretion of pro-inflammatory cytokines IL-1 $\beta$ and IL6 [18]. Additional findings in this study also showed that in tissue taken at time of revision surgery in patients with CoP or CoP implants had clear fibrotic changes [18]. Together these reports present potential immunomodulatory implications of ceramic biomaterials both in combination with other materials such as UHMWPE and alone. Conversely, Bylski et al. [19], reported no significant changes in the expression of TNF $\alpha$ or the pro-osteolytic protein RANK by THP-1 macrophage cells stimulated with alumina particles [19] which therefore demonstrates the paucity in the current literature and brings into question the replicability of the data previously published.

\section{Conclusion}

Ceramic use in hip arthroplasty implants is the most popular alternative to MoP implants and with the projected increase in total hip replacement demand, it is imperative that we take into account the mechanical and biological mechanisms which drive implant failure, in order to improve implant longevity for our patients to reduce the need for revision surgery.

\section{References}

1. Glyn Jones S, Palmer AJ, Agricola R, Price AJ, Vincent TL, et al. (2015) Osteoarthritis. Lancet 386(9991): 376-387.

2. (2020) National Joint Registry. National Joint Registry for England and Wales $17^{\text {th }}$ Annual Report 2020.

3. Evans JT, Evans JP, Walker RW, Blom AW, Whitehouse MR, et al. (2019) How long does a hip replacement last? A systematic review and metaanalysis of case series and national registry reports with more than 15 years of follow-up. Lancet 393(10172): 647-654.

4. López López JA, Humphriss RL, Beswick AD, Thom HHZ, Hunt LP, et al. (2017) Choice of implant combinations in total hip replacement: systematic review and network meta-analysis. BMJ 359: 4651.
5. Schmalzried TP, Jasty M, Harris WH (1992) Periprosthetic bone loss in total hip arthroplasty. Polyethylene wear debris and the concept of the effective joint space. JBJS 74(6): 849-863.

6. Kurtz SM, Lau E, Baykal D, Springer BD (2017) Outcomes of ceramic bearings after primary total hip arthroplasty in the medicare population. J Arthroplasty 32(3): 743-749.

7. Watters TS, Cardona DM, Menon KS, Vinson EN, Bolognesi MP, et al. (2010) Aseptic lymphocyte-dominated vasculitis-associated lesion: A clinicopathologic review of an underrecognized cause of prosthetic failure. American Journal of Clinical Pathology 134(6): 886-893.

8. Lopes R, Philippeau JM, Passuti N, Gouin F (2012) High rate of ceramic sandwich liner fracture. Clin Orthop Relat Res 470(6): 1705-1710.

9. Morlock MM, Witt F, Bishop N, Behn R, Dalla Pria P, et al. (2014) Wear of a composite ceramic head caused by liner fracture. Orthopedics 37(7): e653-e656.

10. Malem D, Nagy MT, Ghosh S, Shah B (2013) Catastrophic failure of ceramic-on-ceramic total hip arthroplasty presenting as squeaking hip. BMJ case reports 2013: bcr2013008614.

11. Campbell J, Rajaee S, Brien E, Paiement GD (2017) Inflammatory pseudotumor after ceramic-on-ceramic total hip arthroplasty. Arthroplasty Today 3(2): 83-87.

12. Anjum SA, Lawrence H, Holland JP, Kirby JA, Deehan DJ, et al. (2016) Effect of cobalt-mediated toll-like receptor 4 activation on inflammatory responses in endothelial cells. Oncotarget 7(47): 76471-76478.

13. Lawrence H, Mawdesley AE, Holland JP, Kirby JA, Deehan DJ, et al. (2016) Targeting toll-like receptor 4 prevents cobalt-mediated inflammation. Oncotarget 7(7): 7578-7585.

14. Mochida Y, Boehler M, Salzer M, Bauer TW (2001) Debris from failed ceramic-on-ceramic and ceramic-on-polyethylene hip prostheses. Clin Orthop Relat Res (389): 113-125.

15. Germain MA, Hatton A, Williams S, Matthews JB, Stone MH, et al. (2003) Comparison of the cytotoxicity of clinically relevant cobalt-chromium and alumina ceramic wear particles in vitro. Biomaterials 24(3): 469479 .

16. Petit A, Catelas I, Antoniou J, Zukor DJ, Huk OL (2002) Differential apoptotic response of J774 macrophages to alumina and ultra-highmolecular-weight polyethylene particles. J Orthop Res 20(1): 9-15.

17. Sterner T, Schütze N, Saxler G, Jakob F, Rader CP (2004) Effects of clinically relevant alumina ceramic, zirconia ceramic and titanium particles of different sizes and concentrations on TNF-alpha release in a human macrophage cell line. Biomed Tech Berl 49(12): 340-344.

18. Bertrand J, Delfosse D, Mai V, Awiszus F, Harnisch K, et al. (2018) Ceramic prosthesis surfaces induce an inflammatory cell response and fibrotic tissue changes. The Bone \& Joint Journal 100-B(7): 882-890.

19. Bylski D, Wedemeyer C, Xu J, Sterner T, Löer F, et al. (2009) Alumina ceramic particles, in comparison with titanium particles, hardly affect the expression of RANK-, TNF- $\alpha$-, and OPG-mRNA in the THP-1 human monocytic cell line. Journal of Biomedical Materials Research Part A 89A(3):707-716. 